\title{
3000 Comparative Examination of Finishing Tochnical Coils Available in Japan
}

Naosuke Kanenaka, ${ }^{1}$ Hiroaki Sato, ${ }^{1}$ Fumihiro Hiraoka, ${ }^{1}$ Hajime Abe, ${ }^{1}$ Koichi Torihashi, ${ }^{2}$ and Shigeo Sora ${ }^{2}$

Objective: In the final stage of coil embolization, microcatheter kickback often occurs and it may be difficult to fill the remaining space with a coil. To avoid microcatheter kickback and achieve successful embolization, soft type coils such as ED coil-10 Extra Soft type R (EDC-10ES) are frequently used as a finishing coil. We compared six different brands of finishing coils to evaluate the efficacy of EDC-10ES.

Case Presentations: This paper presents a representative case of small cerebral aneurysm treated with only EDC-10 ES coils were presented. Furthermore, to compare the degree of coil softness and microcatheter kickback, we verified the in vitro coil performance of six different brands of finishing coils. The first experiment compared the degree of microcatheter kickback in the final stage of coil embolization and the second experiment tested the softness of the delivery wire.

Conclusion: The results verified that EDC-10 ES has less microcatheter kickback in relation to both the coil and delivery wire, compared to the other finishing coils. Consequently, EDC-10 ES was evaluated as a coil with extremely high softness, allowing stable coil placement in the final stage of embolization.

Keywords > EDC-10 ES type R, extremely soft coil, coil tail straightening phenomenon, delivery wire tip softness

\section{Introduction}

Along with surgical clipping, cerebral aneurysm coil embolization is currently the main treatment for cerebral aneurysm. Various sizes and shapes of bare platinum coils are currently being manufactured for aneurysm embolization depending on the intended purpose: framing, filling and finishing. Surgeons can select from among these coil types depending on the situation.

However, the surgeon often has difficulty in making the most appropriate coil selection when they encountered microcatheter instability that is commonly seen at final stage of embolization.

Also in coil embolization of small aneurysms, because the microcatheter is less stable and the risk of hemorrhagic

${ }^{1}$ Department of Endovascular Stroke Center, Tokyo Metropolitan Police Hospital, Nakano-ku, Tokyo, Japan

${ }^{2}$ Department of Neurosurgery, Tokyo Metropolitan Police Hospital, Nakano-ku, Tokyo, Japan

Received: November 9, 2015; Accepted: February 29, 2016

Corresponding author: Naosuke Kanenaka. Department of Endovascular Stroke Center, Tokyo Metropolitan Police Hospital, 4-22-1 Nakano, Nakano-ku, Tokyo 164-0001, Japan

Email: e6f072@yahoo.co.jp

(C)2016 The Editorial Committee of Journal of Neuroendovascular Therapy. All rights reserved. complications is higher, coil selection is more difficult in many cases. ${ }^{1,2}$ At our institution we frequently use a very soft coil as a finishing coil, the ED coil-10 Extra Soft type R (hereinafter referred to as EDC-10 ES), in these situations.

EDC-10 ES is a coil manufactured in Japan (Kaneka Medix Corporation, Osaka) and is characterized by very high softness and less microcatheter kickback. This allows it to search the remaining space inside the aneurysm and fill this space in the final stage of embolization with a higher Volume embolic ratio (VER). Therefore, since its launch in July 2002, EDC-10 ES has often been used in Japan as a finishing coil.

We compared six different brands of finishing coils to evaluate the efficacy of EDC-10ES, and presented two representative cases of cerebral aneurysm treated with EDC-10 ES coils.

\section{Case Presentation and In Vitro Study}

\section{Case presentation}

The patient is a 40 -year-old male. He had a diagnosis of Subarachnoid hemorrhage due to Basilar artery-superior cerebellar artery bifurcation ruptured aneurysm (Fig. 1). Hunt and Kosnik grade I. ${ }^{3}$ WFNS grade I. (World Federation of Neurogical Surgeons Committee on a Universal Subarachnoid Hemorrhage Grading Scale $\left.{ }^{4}\right)$. The aneurysm 


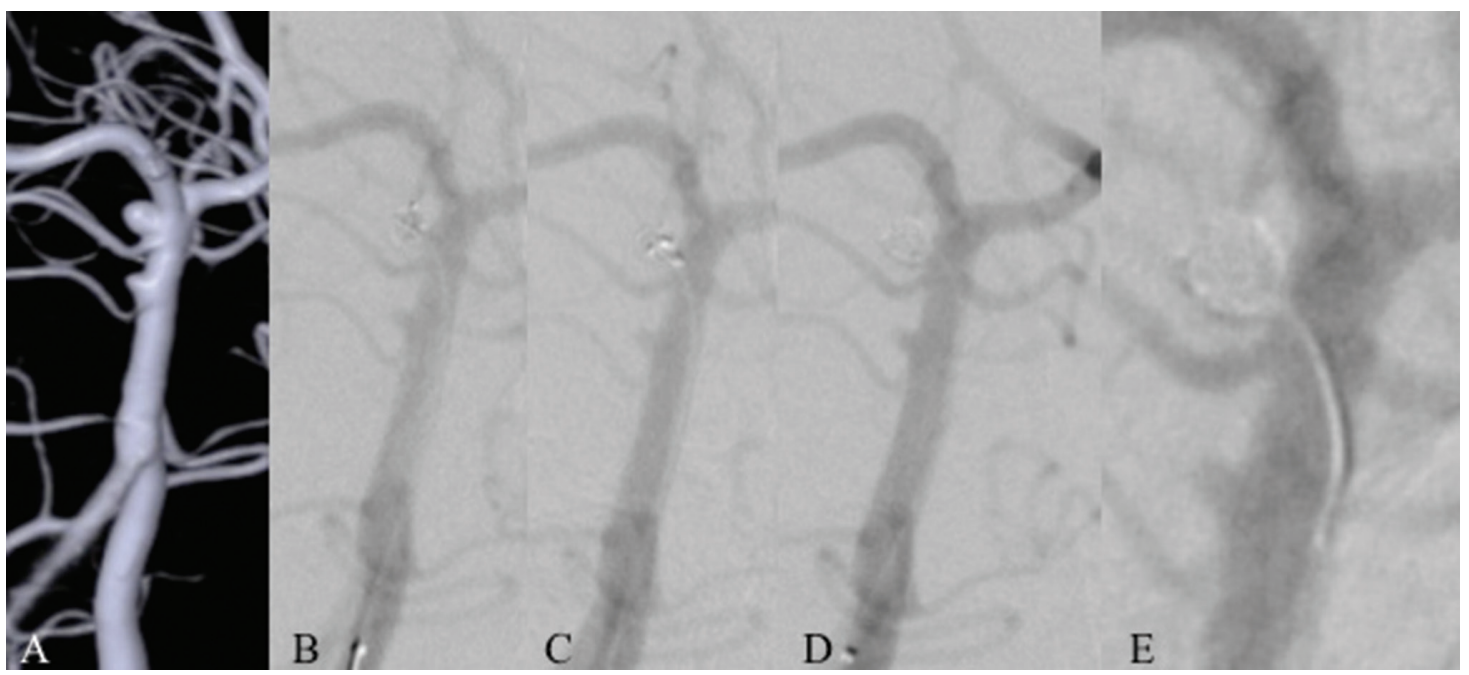

Fig. 1 Rt Basilar artery-superior cerebellar artery bifurcation ruptured aneurysm. (A) Initial 3-dimensional angiogram shows small aneurysm of the basilar artery-superior cerebellar artery. Aneurysm size is $2.9 \mathrm{~mm} \times 2.57 \mathrm{~mm}$. (B) Angiogram shows view after first coil packing; an EDC-10 ES $2.0 \mathrm{~mm} \times 4 \mathrm{~cm}$ coil was used. (C, D) Embolization is performed using another three EDC-10 ES coils $(2.0 \mathrm{~mm} \times 3 \mathrm{~cm}, 2.0 \mathrm{~mm} \times 2 \mathrm{~cm}$ and $1.5 \mathrm{~mm} \times 2 \mathrm{~cm})$. (E) High magnification post-embolization digital subtraction angiogram showed complete obliteration of the aneurysm. EDC-10 ES: ED coil-10 Extra Soft type R

Table 1 Comparison of finishing coils specs: the size is $1.5 \mathrm{~mm} \times 2 \mathrm{~cm}$. The stock wire of ED is not the smallest among these coils. Note that only ED coils use platinum for their stretch-resistant structure, and is same material used for the coils

\begin{tabular}{llccccl} 
Company & Coil brand name & $\begin{array}{c}\text { Secondary } \\
\text { coil }(\mathrm{mm})\end{array}$ & $\begin{array}{c}\text { Length } \\
(\mathrm{cm})\end{array}$ & $\begin{array}{c}\text { Primary } \\
\text { coil (inch) }\end{array}$ & $\begin{array}{c}\text { Stock } \\
\text { wire (inch) }\end{array}$ & SR material \\
Stryker & Target Helical NANO & 1.5 & 2 & 0.01 & 0.00125 & polypropylene \\
Stryker & Target 360 NANO & 1.5 & 2 & 0.01 & 0.00125 & polypropylene \\
MicroVention & HypersoftER & 1.5 & 2 & 0.01 & 0.00125 & polypropylene \\
Codman & Deltaplush & 1.5 & 2 & 0.0096 & 0.0013 & polyglycolic acid \\
Kaneka & ED Extrasoft Type R & 1.5 & 2 & 0.01 & 0.0014 & platinum \\
Covidien & Axium Helix & 1.5 & 2 & 0.0115 & 0.0015 & polypropylene \\
\hline
\end{tabular}

SR: stretch-resistant

was extremely small (The size is $2.9 \mathrm{~mm} \times 2.57 \mathrm{~mm}$ ) and the risk of re-rupture during coil embolization was high. ${ }^{5)}$

We used four coils of all only EDC-10 ES. The embolization was finished with complete obliteration confirmed on cerebral angiography.

\section{In vitro study}

To objectively compare the degree of coil softness and microcatheter kickback, we conducted two experiments to test microcatheter performance using six brands of finishing coil (EDC-10 ES, Target 360 Nano coil [Stryker, Kalamazoo, MI, USA], Target Helical Nano coil [Stryker, Kalamazoo, MI, USA], Axium Helix [ev3 Covidien, Irvine, CA, USA], DeltaPlush [Codman and Shurtleff, Johnson and Johnson, Raynham, MA, USA], HypersoftER [MicroVention TERUMO, Tustin, CA, USA]). All were available in Japan and sized $1.5 \mathrm{~mm} \times$ $2 \mathrm{~cm}$ with the same secondary coil diameter (Table 1).

The first experiment compared the degree of microcatheter kickback in vitro during coil insertion (Fig. 2).
Using a silicone aneurysm model of $1.5 \mathrm{~mm} \times 1.5 \mathrm{~mm}$, coil embolization was performed with six brands of coils (as previously mentioned, unused coils sized $1.5 \mathrm{~mm} \times 2 \mathrm{~cm}$ were used) with the same secondary coil diameter and coil length. To compare the coil embolization status and microcatheter kickback, the coils were inserted manually and, insofar as possible, at the same rate until the detachable part of the coil overlapped the microcatheter tip.

The EDC-10 ES was the only coil where the entire $2 \mathrm{~cm}-$ length could be embolized with negligible microcatheter kickback. When the five other brands were tested, the microcatheter kicked back and deviated to the outside of the aneurysm in the final stage of embolization.

The second experiment, which investigated the influence of the delivery wire system, compared the repelling force of the microcatheter (Fig. 3). Figure 4 shows a comparison of the degree of catheter stretch when the delivery wire was close to the Excelsior SL-10 microcatheter with a tip angle of $90^{\circ}$. The softer the delivery wire, 


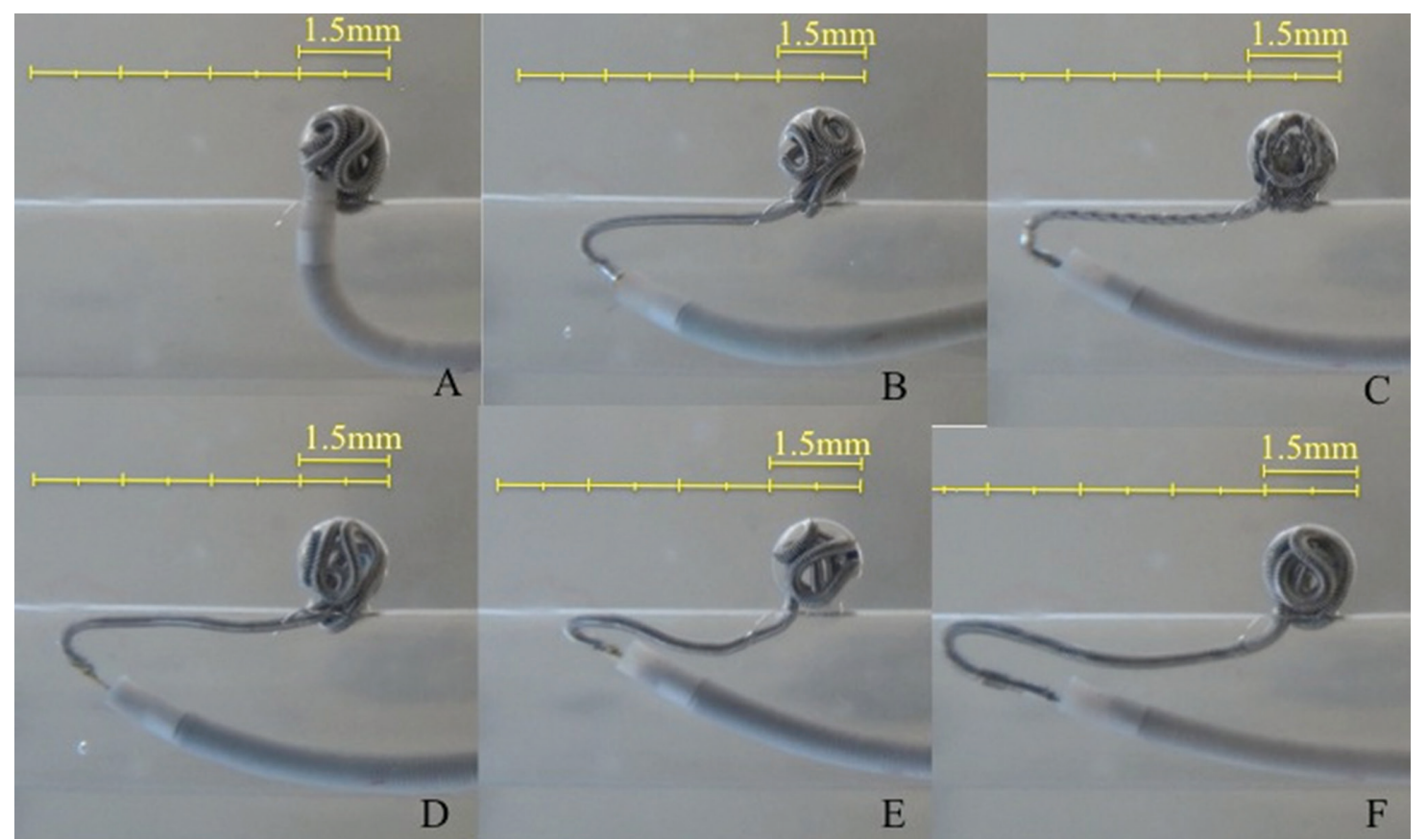

Fig. 2 Comparison of microcatheter kickback phenomenon using a silicone aneurysm model sized $1.5 \mathrm{~mm} \times 1.5 \mathrm{~mm}$. Microcatheter: Excelsior SL-10. (A) EDC-10 ES; (B) HypersoftER; (C) Deltaplush; (D) Target Helical NANO; (E) Target 360 NANO; (F) Axium Helix. EDC-10 ES was the only coil where the entire $2 \mathrm{~cm}$-length could be embolized with negligible microcatheter kickback. When the five other brands were tested, the microcatheter kicked back and deviated to the outside of the aneurysm in the final stage of embolization. EDC-10 ES: ED coil-10 Extra Soft type R

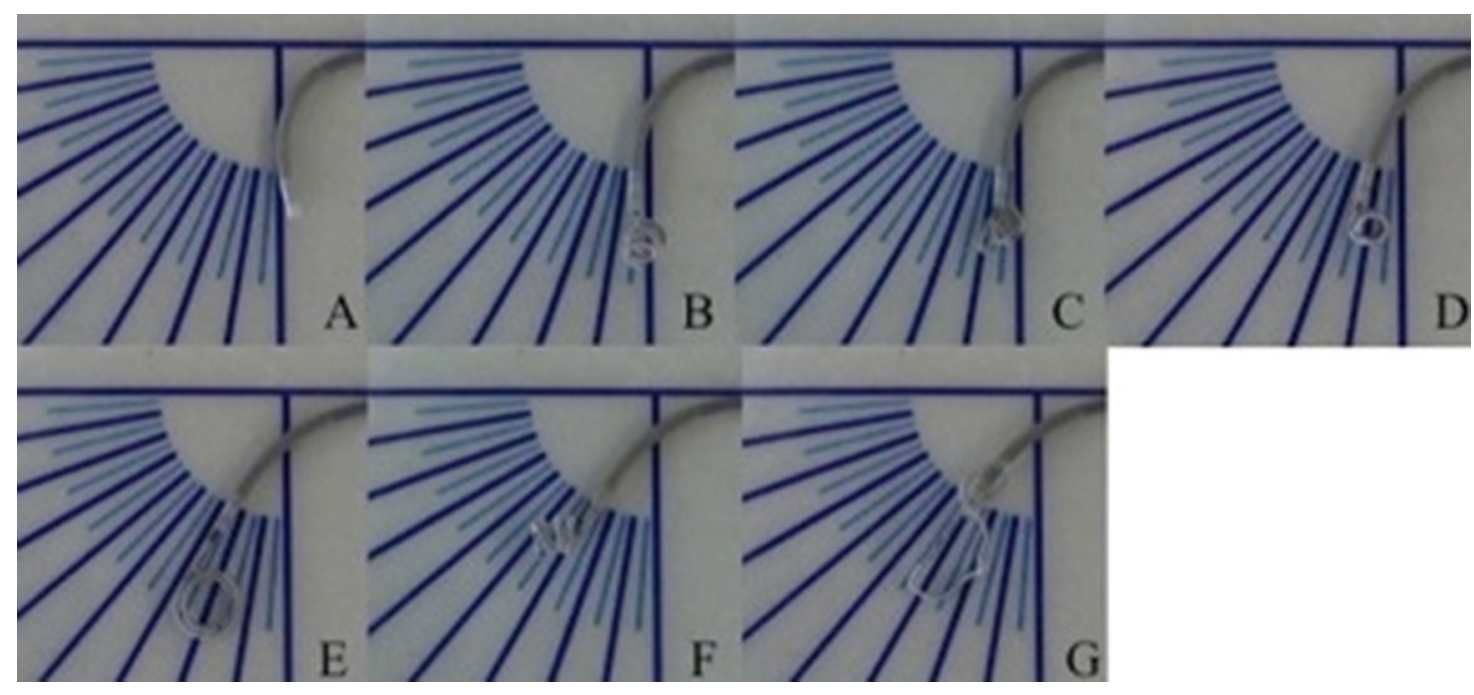

Fig. 3 Comparison of delivery wire tip softness. Microcatheter used an Excelsior SL-10 preshaped 90. (A) Control; (B) HypersoftER; (C) EDC-10 ES; (D) Axium Helix; (E) Target helical NANO; (F) Deltaplush; (G) Target 360 NANO. Microcatheter rebound was very small with HypersoftER and EDC-10 ES, with HypersoftER being slightly superior to EDC-10 ES. The rebound was greater with the other coils (Axium Helix, Target Helical Nano, DeltaPlush and Target 360 Nano ranked in order from greater to smaller rebound). EDC-10 ES: ED coil-10 Extra Soft type R

the lower the degree of microcatheter rebound: the high softness appears to contribute to catheter stability during embolization.

The results showed that there was only very slight microcatheter rebound with HypersoftER and EDC-10 ES, with HypersoftER being slightly superior to EDC-10 ES. The rebound was greater with the other coils (Axium Helix,
Target Helical Nano, DeltaPlush and Target 360 Nano ranked in order from greater to smaller rebound).

\section{Discussion}

Ideally, the objective when using a finishing coil in the final stage of embolization is to be able to place the coil in 
A.

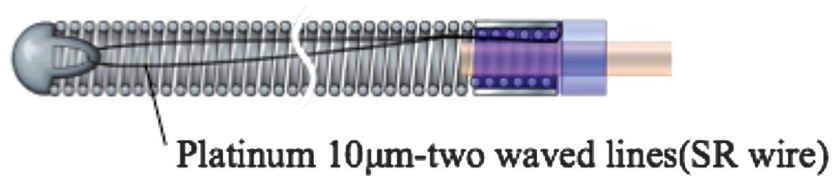

B. PVA

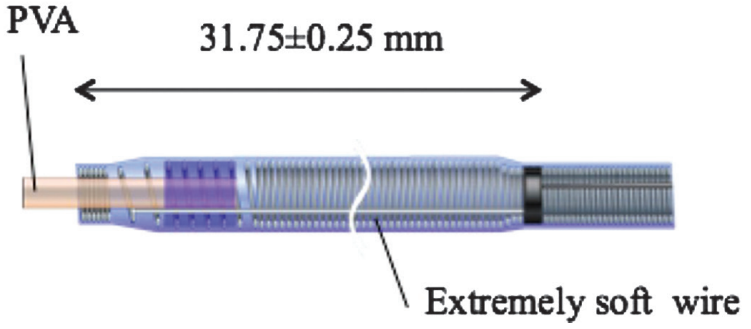

Fig. 4 Shapes of delivery wire tip and structure of stretch resistance (SR). (A) The SR wire is more flexible within the inner lumen of the primary coil because the use of platinum allows the wire to be very thin $(10 \mu \mathrm{m})$. (B) The softest material is used for the part covering $30 \mathrm{~mm}$ of the tip. This appears to lower the repelling force of the delivery wire and reduces the stretch of the microcatheter.

a limited open space and increase VER. At this final stage, however, the detachable part of the coil cannot always be delivered due to microcatheter kickback.

Microcatheter kickback, which may be related to and influenced by vascular diameter and tortuosity, is mainly the result of coil and delivery wire factors. The main coil-related factors are suspected to be both the diameter of the stock wire of the bare platinum coil and the stretch-resistant (SR) structure. ${ }^{6}$

To make a basic bare platinum coil, a stock wire made of an alloy of platinum and tungsten is wound to make a primary coil, and then the primary coil is rewound to make a secondary coil. The diameter of the secondary coil is used when selecting the appropriate coil size for a procedure. The softness of the coil is considered to affect its stiffness and the degree of coil straightening, especially near the coil tail, which is affected to a significant extent by the stretch-resistant structure. The lower the stiffness of the coil is, the more easily the coil can be folded, and the lower the load on the surface contacting the coil.

It has also been reported in the literature that the coil stiffness (i.e. the force applied to the aneurysm wall by the first coil as well as the force applied to the aneurysm wall and coil bundle by the second and subsequent coils) generally depends on the stock wire diameter and is less affected by the diameter of the secondary coil.7) In other words, the smaller the force applied to the wall, the smaller the force producing kickback of the microcatheter.

Moreover, where the remaining space is small in the final stage of embolization, the diameter of the remaining space is often smaller than the secondary coil diameter, and the gap causes coil tail straightening, a phenomenon characteristic of the SR structure, leading to microcatheter kickback. ${ }^{8,9)}$

On the other hand, delivery wire factors are also involved in microcatheter kickback. Action-reaction law ("for every action, there is an equal and opposite reaction") the microcatheter is kicked back when the delivery wire is inserted into the microcatheter. Therefore, the materials and softness of the main body and the tip of the delivery wire as well as the materials of the detachable part of the coil can be significant factors. ${ }^{10)}$

Based on the results of these in vitro experiments, both coil and delivery wire factors appear to be involved in the microcatheter kickback phenomenon. It is considered that the combination of these factors enables EDC-10 ES to achieve coil embolization with a stable microcatheter in the final stage.

Furthermore, when the EDC-10 ES is examined in detail, it is found that the stock wire diameter of EDC-10 ES is 0.0014 inches. Because this is greater than the diameter of 0.00125 inches for Target 360 Nano, HypersoftER and Target 
Helical Nano, the coil stiffness of EDC-10 ES was expected to be higher (Table 1). However, EDC-10 ES has a distinctive SR structure and the SR wire material is platinum, the same material as that of the coil. The corresponding material used in many other coils is polypropylene (PP) or polyglycolic acid (PGA). The SR wire is more flexible within the inner lumen of the primary coil because the use of platinum allows the wire to be very thin $(10 \mu \mathrm{m})$ (Fig. 4A). The corresponding material used in many other coils may be estimated to exceed $40 \mu \mathrm{m}$. This is likely to reduce coil tail straightening in the final stage of embolization.

On the other hand, the delivery wire of EDC-10 ES, which is made of stainless steel and with an effective length of $187 \mathrm{~cm}$, uses a monopolar system with the detachable part made of poly vinyl alcohol (PVA). This structure makes the delivery wire thin and unbreakable. In addition, the softest material is used for the part covering $30 \mathrm{~mm}$ of the tip (Fig. 4B). This appears to lower the repelling force of the delivery wire and reduces the stretch of the microcatheter.

It was considered that a favorable balance of these factors gives the EDC-10 ES coil the characteristics that allow it to search the remaining space inside the aneurysm and fill this space in at the final stage of embolization, contributing to a high VER and making it useful as a finishing coil. This is considered to be why EDC-10 ES achieved satisfactory embolization, while contributing to reduced microcatheter kickback, in the situation where the microcatheter was unstable, as in the case presented here.

\section{Conclusion}

In the situation where the microcatheter is unstable, the usefulness of EDC-10 ES in coil embolization was clearly illustrated by the in vitro study and representative case of small cerebral aneurysm EDC-10 ES has less microcatheter kickback compared to the other finishing coils. Consequently, EDC-10 ES was evaluated as a coil with extremely high softness, which allows stable coil placement in the final stage of embolization.

\section{Disclosure Statement}

The authors declare no conflict of interest associated with this manuscript.

\section{References}

1) van Rooij WJ, Keeren GJ, Peluso JP, et al: Clinical and angiographic results of coiling of 196 very small $(<$ or $=3 \mathrm{~mm}$ ) intracranial aneurysms. AJNR Am J Neuroradiol 2009; 30: 835-839.

2) Yamaura I, Uramoto $S$, Kidera M, et al: Coil embolization of small aneurysms with a maximum diameter of $3 \mathrm{~mm}$. JNET 2010; 4: 99-105.

3) Hunt WE, Kosnik EJ: Timing and perioperative care in intracranial aneurysm surgery. Clin Neurosurg 1974; 21: 79-89.

4) Report of World Federation of Neurological Surgeons Committee on a Universal Subarachnoid Hemorrhage Grading Scale. J Neurosurg 1988; 68: 985-986.

5) Ioannidis I, Lalloo S, Corkill R, et al: Endovascular treatment of very small intracranial aneurysms. J Neurosurg 2010; 112: 551-556.

6) Matsubara N, Miyachi S, Nagano Y, et al: Experimental study of generation pattern of coil insertion force using an force sensor system: investigation of friction state between coil and aneurysm wall determined by difference of coil insertion method and insertion speed. JNET 2010; 4: 84-90.

7) Ohashi M, Nomura H, Kyoshima K, et al: Aneurysmal wall stress caused by platinum coils is not dependent on the size of a coil but on a diameter of the stock wire: in-vitro study. JNET 2013; 7: 81-87.

8) Miyachi S, Izumi T, Matsubara N, et al: The mechanism of catheter kickback in the final stage of coil embolization for aneurysms: the straightening phenomenon. Interv Neuroradiol 2010; 16: 353-360.

9) Izumi $T$, Miyachi S, Matsubara $N$, et al: Phenomenon of straitening at proximal end of the coil during parent artery occlusion for the internal carotid artery aneurysm: case report. JNET 2011; 5: 173-176.

10) Koyama J, Hanaoka Y, Sato A, et al: Characteristic features of coil delivery wires for cerebral aneurysm embolization. JNET 2014; 8: 21-25. 